\title{
Protocol for systematic reviews of determinants/ correlates of obesity-related dietary and physical activity behaviors in young children (preschool 0 to 6 years): evidence mapping and syntheses
}

Rajalakshmi Lakshman ${ }^{1,2^{*}}$, Veena Mazarello Paes ${ }^{3}$, Kathryn Hesketh ${ }^{2}$, Claire O'Malley ${ }^{4}$, Helen Moore ${ }^{4}, K^{\prime}$. Ong ${ }^{1,2}$, Simon Griffin ${ }^{1,2}$, Esther van Sluijs ${ }^{1,2}$ and Carolyn Summerbell ${ }^{4}$

\begin{abstract}
Background: The aim of these reviews is to inform the design and content of interventions to reduce obesity in young children. The behaviors that are associated with obesity/overweight have been studied extensively; however, the factors associated with these behaviors in young children ( 0 to 6 years) have not been systematically reviewed. Over the past few years the focus of obesity prevention has shifted to preschool children because of the high prevalence of obesity at school entry and recognition that habits formed in early life could track into adulthood. In order to develop effective interventions and change behavior, it is important to understand the factors that are associated with those behaviors. For example, we need to understand whether it would be more important to target the family, childcare settings or the wider environment and identify the most effective way of changing these energy balance related behaviors.
\end{abstract}

Methods/Design: Quantitative (intervention and observational) and qualitative literature on determinants/correlates of fruit and vegetable intake, sugar sweetened beverage and other unhealthy diet intake, and physical activity and sedentary behaviors in young children will be systematically identified, mapped and reviewed. A common search strategy (no language or period restrictions) will be used to identify papers from eight electronic databases and this will be supplemented by hand-searching. Next, studies in developed countries that examine the factors associated with these behaviors in children aged 0 to 6 years (at baseline) will be screened and mapped descriptively followed by in-depth data extraction, quality assessment and synthesis. Data from quantitative studies will be summarized using either forest plots or harvest plots and narrative synthesis, and qualitative studies using thematic analysis. Qualitative evidence will be integrated with the quantitative evidence, using a parallel synthesis approach, to provide a deeper understanding of effective strategies to influence these energy balance related behaviors.

Discussion: In addition to updating and mapping current research, these reviews will be the first to comprehensively synthesize and integrate both the quantitative and qualitative evidence pertaining to determinants/correlates/barriers/facilitators of obesity related behaviors in this young age group ( 0 to 6 years) with the aim of informing future interventions.

Trial registration: International Prospective Register for Systematic Reviews (PROSPERO) Registration number: CRD42012002881

\footnotetext{
* Correspondence: rl284@mrc-epid.cam.ac.uk

${ }^{1}$ MRC Epidemiology Unit, Addenbrookes Hospital, Box 285, Cambridge CB2

OQQ, UK

${ }^{2}$ UKCRC Centre for Diet and Activity Research (CEDAR), Institute of Public

Health, Cambridge, Box 296, CB2 OSR, UK

Full list of author information is available at the end of the article
} 


\section{Background}

The prevalence of childhood overweight and obesity has increased 2- to 4-fold between 1980 and 2000 in developed countries [1], although levels have started to plateau [2-4]. Obesity is increasing even in preschool children and, in 2010, 43 million preschool children (35 million in developing countries) were overweight or obese [5]. The rising prevalence of childhood obesity therefore presents a major public health challenge for the 21st century, increasing the burden of chronic non-communicable diseases in both developed and developing countries [6].

The preschool years are a period of rapid growth and habit formation. Hence, it is possible that at least some of the solutions to the obesity epidemic will be found here, yet few interventions have been developed for preschool children. Recent systematic reviews of obesity prevention interventions in preschool children have concluded that the evidence base for interventions in this age group was sparse [7,8]. The 2011 updated Cochrane review on interventions for preventing obesity in children included 55 studies, only eight of which were in children aged 0 to 5 years, yet these studies showed the largest intervention effects [9].

In the simplest sense, obesity is an imbalance between energy intake and energy expenditure over a long period of time. At an individual level, behavior is key in influencing energy intake (mainly diet) and energy expenditure (mainly physical activity). It is therefore important to understand the behaviors that lead to obesity and the factors that are associated with such behaviors. Interventions to improve health-related behaviors targeting the most important determinants/correlates of these behaviors are more likely to be effective $[10,11]$. The socioecological model [12] provides a useful framework for defining the level of determinant or correlate: individual (for example, gender, ethnicity); family (for example, parenting style, single parent, siblings); childcare setting/ preschool (for example, school policies); community/ neighborhood (for example, food outlets, parks, safety); and policy/media/wider (for example, campaigns, taxation). Use of this framework will allow us to understand whether it would be more important to target the family, childcare settings or the wider environment and what would be the most effective way of doing this (see Figure 1 for schematic diagram outlining the scope of the review).

A number of systematic reviews have been conducted examining the effects of diet and physical activity on the development of obesity and chronic diseases; however, few reviews have focused on the more upstream determinants/correlates of these behaviors in young children. Where such reviews have been conducted, the results have been inconsistent due to different inclusion criteria (age range, study period, study populations) [13-15] and no review to date has attempted to combine observational, intervention and qualitative evidence to provide a comprehensive overview of the literature on determinants/correlates of obesogenic behaviors in children aged 0 to 6 years.

Hence this work aims to comprehensively review the quantitative (observational and intervention) and qualitative literature on determinants/correlates of fruit and vegetable intake, sugar-sweetened beverage and other unhealthy diet intake, and physical activity and sedentary behaviors in preschool children. Secondary questions include: 1) which of these determinants/correlates are modifiable and can therefore be addressed through interventions; 2) should interventions be focused at the individual, family, childcare setting, community or policy level; and 3) what are the gaps in the existing literature and areas for future research?

\section{Methods/Design}

The methods follow established criteria for the rigorous conduct and reporting of systematic reviews [16]. For each of the three topic areas (fruit and vegetable intake, sugar sweetened beverages and unhealthy diet intake, and physical activity and sedentary behavior) the reviews will be carried out in three stages as described in the Evidence for Policy and Practice Information (EPPI) and coordinating centre reviews on barriers and facilitators to fruit and vegetable intake and physical activity in 4- to 10-year-old children $[17,18]$.

Stage 1 involves searching of bibliographic databases, application of broad inclusion and exclusion criteria. and synthesis of a descriptive map of the number, types and quality attributes of existing research studies.

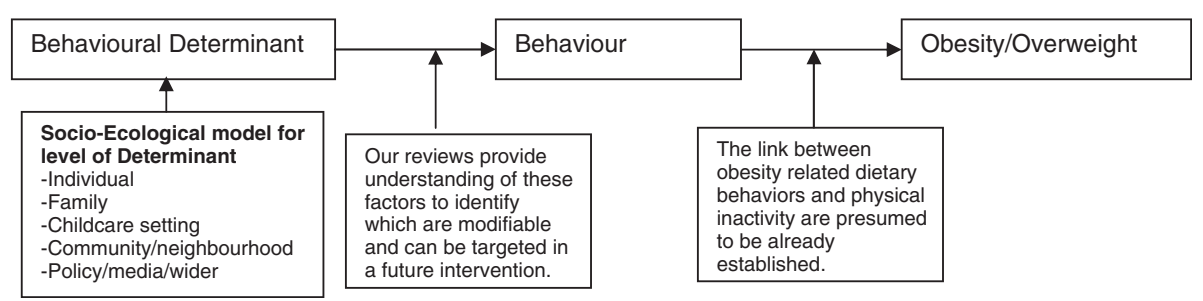

Figure 1 Schematic diagram showing focus of review. 
Stage 2 will take a subset of high-quality quantitative studies identified in Stage1 for detailed data extraction and in-depth synthesis.

Stage 3 will involve a thematic analysis of the qualitative literature to be integrated with the findings of the quantitative literature using a parallel synthesis approach as used previously [19]. Using the evidence from the qualitative research to explain the quantitative findings will provide a deeper understanding of effective strategies to influence these energy balance related behaviors.

\section{Search strategy}

As many studies are common to the three review topics, the database searches, processes of developing criteria for including studies and identifying and classifying studies were run in tandem for all the reviews (by HM). A common search strategy was used to identify papers from eight electronic databases - Medline, Embase (via OVID), Cinhal, Psychinfo (via Ebsco), Web of Knowledge (via Thomson Reuters), British Nursing Index (BNI), Applied Social Sciences Index and Abstracts (ASSIA) and Sociological Abstracts (via Proquest).

A number of initial scoping searches were carried out to refine the search strategy to maximize sensitivity and specificity. This included contacting experts in the field and identifying key publications followed by running the searches and ensuring that important publications were captured. The four sets of search terms relate to the population (young children), exposure (terms to capture observational, intervention, qualitative studies and review articles), outcome (diet and physical activity) and to exclude clinical populations (see Additional file 1 for search strategy). Since previous reviews showed that restricting searches to certain time periods resulted in conflicting conclusions [13], no time period or language restrictions were applied. All identified articles were imported into an Endnote Database and de-duplicated. This will be supplemented by hand-searching references of included articles and relevant reviews.

\section{Inclusion criteria}

Observational (non-intervention) longitudinal (prospective and retrospective) and intervention (randomized control trials (RCTs) and non-RCTs) studies that quantify the association between a risk factor/correlate/determinant AND obesity-related dietary behavior or physical activity or sedentary behavior in children aged 0 to 6 years at baseline will be included. Objectively (diet diaries, food records, 24-hour recalls, accelerometers, combined heart-rate monitors and pedometers) and subjectively (self-report, questionnaires) measured outcomes will be included. Qualitative studies that provide greater in-depth understanding of barriers and facilitators of these behaviors will also be included. Studies of obese participants will be included. Only studies that focus on investigating correlates (cross-sectional association) or determinants (prospective association) of the relevant behaviors will be included, and studies that merely present a descriptive table in an otherwise unrelated study will not be included.

\section{Exclusion criteria}

The following are exclusion criteria: non-human studies; cross-sectional studies (only to be included in absence of other high-level evidence); laboratory-based studies (such as the vitamin and preloading diets); studies on health outcomes for these behaviors (for example, studies describing the association between sedentary behavior and obesity or cardiovascular risk factors); quantitative studies that measure these behaviors but do not describe an association with any other variables; studies in clinical populations (for example, malnutrition, disability, allergy, dental caries, asthma, cerebral palsy, cystic fibrosis, autism etc.); studies on breast/bottle feeding and weaning of infants.

\section{Study selection procedure}

Three reviewers $(\mathrm{KH}, \mathrm{CO}, \mathrm{VP})$ will undertake title and abstract screening in small batches $(n=500)$ based on a piloted screening protocol. One senior reviewer (CS) will screen the same studies and compare results until there is less than 5\% discrepancy, after which screening will be done individually [20]. An overall $10 \%$ of the total articles will be randomly selected and double screened by two additional reviewers (RL, EvS). Papers which meet the inclusion criteria will be ordered for full review. Specific study details such as study design, country of study, study population, exposures assessed, outcome assessed and other valuable information will be extracted to an IN/OUT spreadsheet/form. At this stage, cross-sectional studies will not be excluded. Based on this information, an evidence map of the existing literature will be created (stage 1). Any disagreement will be resolved by discussion and re-examination of the article. All studies meeting the inclusion criteria will be sorted for further review and data extraction, according to the research question or the behavior being investigated.

\section{Evidence mapping}

A descriptive map of the evidence will be created to highlight gaps in the evidence base, but also to identify where sufficient data exist to warrant a review. Data will be synthesized on countries the studies came from, publication year, study design (for example, cross-sectional, longitudinal, intervention, qualitative), behavior studied (for example, fruit and vegetable intake, sugar-sweetened beverage or other unhealthy diet intake, physical activity or sedentary behavior) and type and level of determinant 
studied (for example, individual, family, childcare, community or wider).

\section{Data extraction}

Three reviewers (KH, CO, VP) will systematically review the studies pertaining to the allocated research question or behavior. A data extraction form/spreadsheet has been piloted to ensure consistency of data extraction across reviews and reviewers. Data will be extracted into the spreadsheet by one reviewer and a proportion of the studies will be double-reviewed by a second reviewer (RL, EvS, CS). Discrepancies will be resolved by discussion within the review team. For longitudinal studies (observational or intervention), the latest data available before the children are 6 years old will be included. The data extraction form will be organized to specify the level of determinant - individual, family, childcare, community or wider (See Additional file 2 for details of data extracted).

\section{Quality assessment}

Study quality will be systematically assessed against preset quality criteria using standard quality assessment tools specified for the respective study designs and used by the EPPI centre as follows.

\section{Intervention studies}

The following criteria will be assessed in intervention studies: randomization, effect of intervention reported for all outcomes, pre-intervention data on all outcomes, post-intervention data on all outcomes, allocation concealment, blinding, objective measurement of outcome, and retention $>70 \%$.

\section{Non-intervention studies}

For non-intervention studies we will be looking at the following criteria: number of participants, representativeness/generalizability, prospective data collection (versus cross-sectional), multivariate analyses (versus univariate), objective (versus subjective) measure of exposure, and objective measure of outcome.

\section{Qualitative studies}

In qualitative studies, the following set criteria will be investigated: research questions clearly stated, approach appropriate for the research question, qualitative approach clearly justified, study context clearly described, role of the researcher clearly described, sampling method clearly described, sampling strategy appropriate for the research question, method of data collection clearly described, data collection method appropriate, method of analysis clearly described, analysis appropriate for the research question, and conclusions supported by sufficient evidence.

\section{Data synthesis}

Narrative and, where possible, statistical data synthesis will be undertaken.

\section{Intervention studies}

Where possible, an attempt will be made to meta-analyze the data and present results as a Forest-plot [21], only including randomized trials of high-quality studies with a low risk of bias.

\section{Non-intervention studies}

Where it is not possible to synthesize the data using Forest plots, Harvest plots [22] will be used. Direction and strength of the association will be summarized using the following symbols: significant negative association, - -; non-significant negative association, -; null association, 0; non-significant positive association, +; significant positive association, ++ . Results for categorical and continuous outcome variables will be consistently recoded so that a single or double + always denotes higher risk for the undesirable behavior, and a single or double - always denotes a lower risk for the undesirable behavior. These data will then be displayed using bar charts as follows:

1. Position based on direction and strength of association $(++,+, 0,-,--)$.

2. Height of bar representing size of study.

3. Color of bar representing quality: black, dark grey and light grey with darker bars representing higher quality studies.

4. Symbol on top for study identification.

Conclusions will be drawn based on the consistency of results of studies of the highest available quality level, consistency defined as $>75 \%$ of results being in the same direction [23].

\section{Qualitative studies}

Thematic synthesis will be used to summarize qualitative studies. The qualitative findings will be integrated with the quantitative findings using the parallel synthesis approach recommended for mixed-methods research synthesis [17]. Themes identified in the qualitative studies will be used to interpret the findings of the quantitative studies and recommendations will be drawn for future interventions. For example, a review of barriers and facilitators to fruit and vegetable intake in 4- to 10-year-old children concluded that, although the qualitative evidence suggested that branding fruit and vegetables as 'tasty' rather than 'healthy' and making the messages salient to children and the social context were important, few interventions used these strategies [17]. 


\section{Discussion}

As far as we are aware, these reviews will be the first to integrate, in a rigorous and systematic way, the findings of quantitative (both observational and intervention studies) and qualitative research on this topic. It is considered that this type of synthesis provides a more complete and trustworthy picture than relying on syntheses of any one type of research in isolation [17]. After de-duplication, 37,868 papers were downloaded to an Endnote database (Medline, $\mathrm{n}=$ 20374; Embase, $\mathrm{n}=17331$; Cinahl, $\mathrm{n}=775$; Psychinfo, $\mathrm{n}=1868$; Sociological Abstracts, $\mathrm{n}=135$; ASSIA, $\mathrm{n}=113$; Web of Knowledge, $\mathrm{n}=13455$; and BNI, $\mathrm{n}=291$ ). Figure 2 demonstrates a flow diagram of the study selection process.

\section{Comparison with previous reviews in young children}

Most reviews have examined obesity prevention interventions. A Health Technology Assessment (HTA) systematic review in 2009 [7] and a 2010 systematic review [8] found an absence of effective interventions to prevent obesity in preschool children. The HTA review only included studies that reported body mass index (BMI) as an outcome and identified three studies. The 2010 review included studies reporting BMI, diet or physical activity as outcomes and identified 23 studies. Both reviews concluded that the evidence base for obesity prevention in preschool children was sparse and further research was urgently needed with well designed RCTs in preschool children. More recently, the ToyBox Study Group have published a series of systematic reviews relating to childcare-based (preschools/schools) interventions to prevent obesity in children aged 4 to 6 years [24]. Those reviews included educational strategies [25], psychological approaches [26] and behavioral models [27] underpinning interventions targeting diet and physical activity in the preschool/school setting, and recommended that childhood obesity was not an issue for the education sector alone, but needed to be tackled at a multi-sectoral level [28]. Our reviews on the behavioral determinants/correlates will provide further evidence on the levels (individual, family, preschool, community or wider) at which interventions could be effectively targeted.

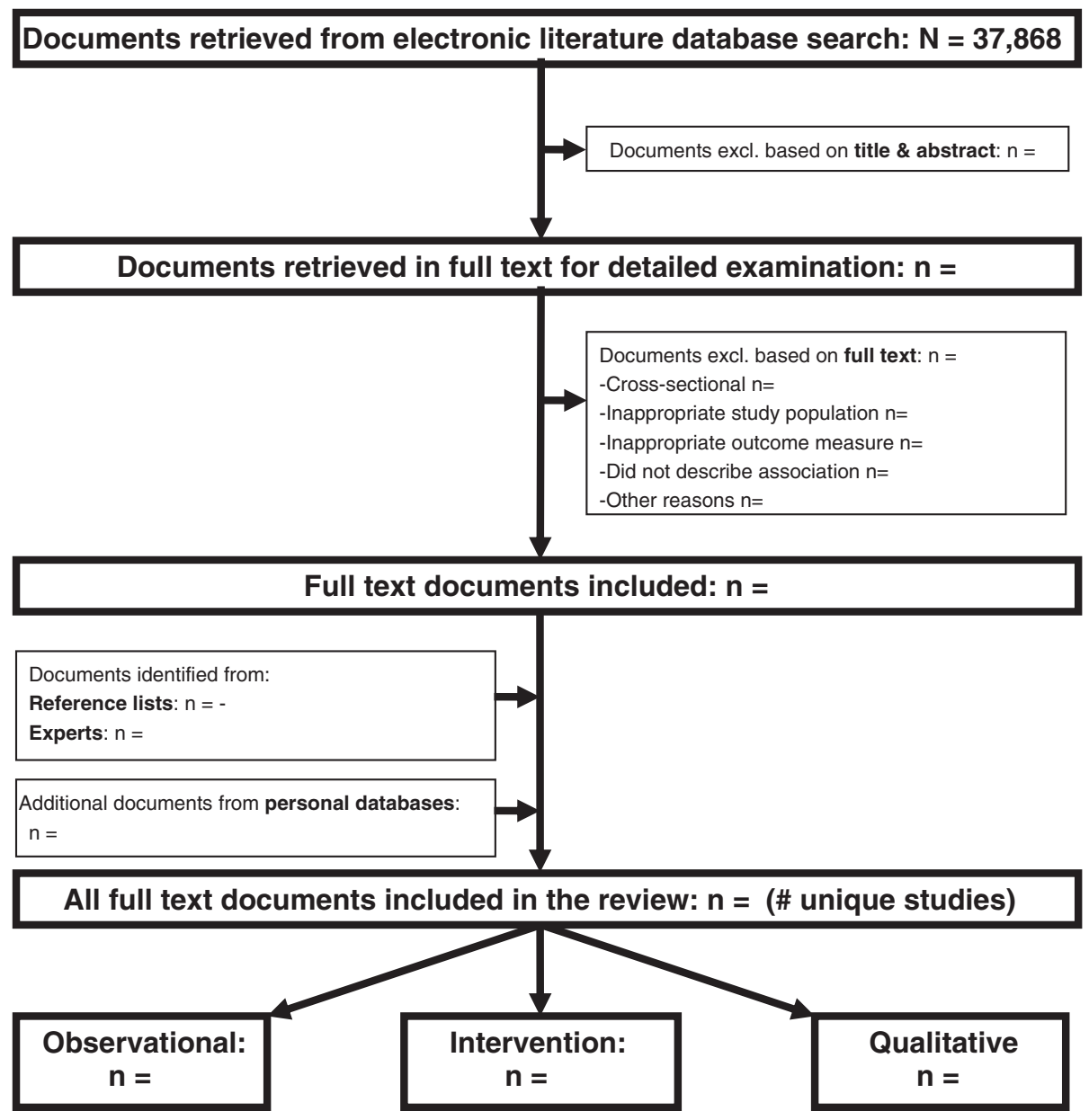

Figure 2 Flowchart presenting an overview of the search results. 
Previous systematic reviews that examined the correlates of physical activity in 2008 [15] and sedentary behavior in 2010 [14] in 2- to 5-year-old children found that male sex, having active parents and spending more time outdoors was associated with higher physical activity, but the evidence for sedentary behavior was inconclusive. A more recent systematic review in 2011 [13] of correlates of energy balance-related behaviors in 4- to 6-year-old children concluded that gender, age and socioeconomic status were not associated with physical activity, while an indeterminate result was found for ethnicity. Gender and ethnicity were not associated with sedentary behavior and indeterminate results were found for age and socioeconomic status. Watching television was associated with a higher consumption of snacks and sugar-sweet beverages [13].

There are no systematic reviews of correlates of dietary behaviors specifically in 0 - to 6-year-old children. We reviewed the quantitative evidence on determinants of early weaning and included 72 studies that examined 43 determinants; however, only six determinants were consistently associated with early weaning (young maternal age, low maternal education, low socio-economic status, absence or short duration of breastfeeding, maternal smoking, and lack of information and advice from healthcare providers) [23]. A review of the determinants of fruit and vegetable consumption among children and adolescents identified 98 papers and found that age, gender, socioeconomic position, preferences, parental intake and home availability/accessibility were important determinants/correlates of fruit and vegetable intake for all children under 18 years of age [29]. A review of determinants/correlates of children's eating patterns and diet quality found that physical, social and family environments were important [30].

By including more recent studies, quantitative and qualitative studies, we aim to be able to draw more robust conclusions about the barriers and facilitators to be targeted in future interventions. Also younger children ( 0 to $3 / 4$ years) may be influenced by different factors to those affecting 4- to 6-year-old children and, hence, it is important to study this entire age range. Indeed, there has been a recent increase in intervention studies targeting this younger age group [8,31-35] and it is important to include this age group in these reviews.

\section{Dissemination and plans for updating}

The results will be disseminated to academic and nonacademic audiences through peer-reviewed publications, conferences, formal presentations and in formal meetings.

Currently there are no plans for updating the reviews but this will be considered if a significant amount of new data becomes available.

\section{Additional files}

Additional file 1: Search Strategy.

Additional file 2: Details of data extracted for different study designs.

\section{Abbreviations}

ASSIA: Applied Social Sciences Index and Abstracts; BMI: Body mass index; BNI: British Nursing Index; EPPI: Evidence for Policy and Practice Information; HTA: Health Technology Assessment; PROSPERO: International Prospective Register for Systematic Reviews; RCT: Randomized control trial.

\section{Competing interests}

The authors declare that they have no competing interests.

\section{Authors' contributions}

$\mathrm{RL}$ conceived the study and wrote the first draft. All authors contributed to designing the search strategy, commented on all aspects of the protocol, and approved the final version. HM carried out the database searches. VP, KH and CO carried out title and abstract screening. CS, RL and EvS contributed to quality assurance of the screening process. All authors read and approved the final manuscript.

\section{Acknowledgements}

This is an outline of independent research funded by the National Institute of Health Research, School for Public Health Research (NIHR SPHR). The views expressed are those of the author(s) and not necessarily those of the NHS, the NIHR or the Department of Health. The National Institute for Health Research's School for Public Health Research (NIHR SPHR) is a partnership between the Universities of Sheffield, Bristol, Cambridge, UCL; The London School for Hygiene and Tropical Medicine; The Peninsula College of Medicine and Dentistry; the LiLaC collaboration between the Universities of Liverpool and Lancaster and Fuse; The Centre for Translational Research in Public Health, a collaboration between Newcastle, Durham, Northumbria, Sunderland and Teesside Universities.

\section{Author details}

${ }^{1}$ MRC Epidemiology Unit, Addenbrookes Hospital, Box 285, Cambridge CB2 OQQ, UK. ${ }^{2}$ UKCRC Centre for Diet and Activity Research (CEDAR), Institute of Public Health, Cambridge, Box 296, CB2 OSR, UK. ${ }^{3}$ Cambridge Institute of Public Health, Cambridge CB2 OSR, UK. ${ }^{4}$ Obesity Related Behaviours Research Group, Durham University, Stockton on Tees TS17 6BH, UK.

Received: 4 February 2013 Accepted: 22 April 2013

Published: 10 May 2013

\section{References}

1. Ebbeling CB, Pawlak DB, Ludwig DS: Childhood obesity: public-health crisis, common sense cure. Lancet 2002, 360:473-482.

2. Ogden CL, Carroll MD, Kit BK, Flegal KM: Prevalence of obesity and trends in body mass index among US children and adolescents, 1999-2010. JAMA 2012, 307(5):483-490.

3. Rokholm B, Baker $\mathrm{J}$, Sorensen TIA: The levelling off of the obesity epidemic since the year 1999: a review of evidence and perspectives. Obes Rev 2010, 11:835-846.

4. Rolland-Cachera MF, Peneau S: Stabilization in the prevalence of childhood obesity: a role for early nutrition. Int J Obes 2010, 34:1524-1525.

5. de Onis M, Blossner M, Borghi E: Global prevalence and trends of overweight and obesity among preschool children. Am J Clin Nutr 2010, 92:1257-1264.

6. Lakshman R, Elks CE, Ong KK: Childhood Obesity. Circulation 2012 126:1770-1779.

7. Bond M, Wyatt K, Lloyd J, Welch K, Taylor R: Systematic review of the effectiveness and cost-effectiveness of weight management schemes for the under fives: a short report. Health Technol Assess 2009, 13:1-100.

8. Hesketh KD, Campbell KJ: Interventions to prevent obesity in $0-5$ year olds: an updated systematic review of the literature. Obesity 2010, 18:S27-S35 
9. Waters E, Sanigorski A, Hall BJ, Brown T, Campbell KJ, Gao Y, Armstrong R, Prosser $\mathrm{L}$, Summerbell $\mathrm{C}$ : Interventions for preventing obesity in children. Cochrane Database Syst Rev 2011, 12:1-191.

10. Baranowski T, Cullen KW, Baranowski J: Psychosocial correlates of dietary intake: advancing dietary intervention. Annu Rev Nutr 1999, 19:17-40.

11. Brug J, Oenema A, Ferreira I: Theory, evidence and intervention mapping to improve behavior nutrition and physical activity interventions. Int $J$ Behav Nutr Phys Act 2005, 2:2.

12. McLeroy KR, Bibeau D, Steckler A, Glanz K: An ecological perspective on health promotion programs. Health Educ Q 1988, 15:351-377.

13. De Craemer M, De Decker E, de Bourdeaudhuij I, Vereecken C, Deforche B, Manios Y, Cardon G: Correlates of energy balance-related behaviours in preschool children: a systematic review. Obes Rev 2012, 13(Suppl 1):13-28.

14. Hinkley T, Salmon J, Okely AD, Trost SG: Correlates of sedentary behaviours in preschool children: a review. Int J Behav Nutr Phys Act 2010, 7:66.

15. Hinkley T, Crawford D, Salmon J, Okely AD, Hesketh K: Preschool children and physical activity: a review of correlates. Am J Prev Med 2008, 34:435-441.

16. Centre for Reviews and Dissemination (CRD): Systematic Reviews: CRD's Guidance for Understaking Reviews in Healthcare. 3rd edition. University of York, UK: CRD; 2008

17. Thomas J, Sutcliffe K, Harden A, Oakely A, Oliver S, Rees R, Brunton G, Kavanagh J: Children and Healthy Eating: A Systematic Review of Barriers and Facilitators. London: EPPI-Centre, Social Science Research Unit, Institute of Education, University of London; 2003.

18. Brunton G, Harden A, Rees R, Kavanagh J, Oliver S, Oakely A: Children and Physical Activity: A Systematic Review of Barriers and Facilitators. London: EPPI-Centre, Social Science Research Unit, Institute of Education, University of London; 2003.

19. Barnett I, Guell C, Ogilvie D: The experience of physical activity and the transition to retirement: a systematic review and integrative synthesis of qualitative and quantitative evidence. Int J Behav Nutr Phys Act 2012, 9:97.

20. Bonell C, Harden A, Wells H, Jamal F, Fletcher A, Petticrew M, Thomas J, Campbell R, Murphy S, Moore L: Protocol for a systematic review of the effects of schools and school-environment interventions on health: evidence mapping and syntheses. BMC Public Health 2011, 11:453.

21. Pace NL: Research methods for meta-analyses. Best Pract Res Clin Anaesthesiol 2011, 25:523-533.

22. Ogilvie D, Fayter D, Petticrew M, Sowden A, Thomas S, Whitehead M, Worthy G: The harvest plot: a method for synthesising evidence about the differential effects of interventions. BMC Med Res Methodol 2008, 8:1-7.

23. Wijndaele K, Lakshman R, Landsbaugh JR, Ong KK, Ogilvie D: Determinants of early weaning and use of unmodified cow's milk in infants: a systematic review. J Am Diet Assoc 2009, 109:2017-2028.

24. Manios Y, Grammatikaki E, Androutsos O, Chinapaw MJM, Gibson EL, Buijs G, lotova V, Socha P, Annemans L, Wildgruber A, Mouratidou T, Yngve A, Dunvinage $K$, de Bourdeaudhuji I: A systematic approach for the development of a kindergarten-based intervention for the prevention of obesity in preschool age children: the ToyBox-study. Obes Rev 2012, 13:3-12.

25. Kreichauf S, Wildgruber A, Krombholz H, Gibson EL, Vogele C, Nixon CA, Douthwaite W, Moore H, Manios Y, Summerbell CD: Critical narrative review to identify educational strategies promoting physical activity in preschool. Obes Rev 2012, 13:96-105.

26. Gibson EL, Kreichauf S, Wildgruber A, Vogele C, Summerbell CD, Nixon C, Moore H, Douthwaite W, Manios Y: A narrative review of psychological and educational strategies applied to young children's eating behaviours aimed at reducing obesity risk. Obes Rev 2012, 13(Suppl 1):85-95.

27. Nixon CA, Moore HJ, Douthwaite W, Gibson EL, Vogele C, Kreichauf S, Wildgruber A, Manios Y, Summerbell C: Identifying effective behavioural models and behaviour change strategies underpinning preschool- and school-based obesity prevention interventions aimed at 4-6-year-olds: a systematic review. Obes Rev 2012, 13(Suppl 1):106-117.

28. Summerbell CD, Moore HJ, Vogele C, Kreichauf $S$, Wildgruber A, Manios $Y$, Douthwaite W, Nixon C, Gibson E: Evidence-based recommendations for the development of obesity prevention programs targeted at preschool children. Obes Rev 2012, 13:129-132.

29. Rasmussen M, Krolner R, Klepp Kl, Lytle L, Brug J, Bere E, Due P: Determinants of fruit and vegetable consumption among children and adolescents: a review of the literature. Part I: quantitative studies. Int J Behav Nutr Phys Act 2006, 3:22.
30. Patrick H, Nicklas TA: A review of family and social determinants of children's eating patterns and diet quality. J Am Coll Nutr 2005, 24:83-92

31. Askie L, Baur L, Campbell K, Daniels L, Hesketh K, Magarey A, Mihrshahi S, Rissel C, Simes J, Taylor B, Taylor R, Voysey M, Wen L: Study protocol: the Early Prevention of Obesity in CHildren (EPOCH) Collaboration - an individual patient data prospective meta-analysis. BMC Public Health 2010, 10:728.

32. Daniels L, Magarey A, Battistutta D, Nicholson J, Farrell A, Davidson G, Cleghorn G: The NOURISH randomised control trial: positive feeding practices and food preferences in early childhood - a primary prevention program for childhood obesity. BMC Public Health 2009, 9:387.

33. Lanigan J, Barber S, Singhal A: Prevention of obesity in preschool children. Proc Nutr Soc 2010, 69:204-210.

34. Rudolf MC, Hunt C, George J, Hajibagheri K, Blair M: HENRY: development, pilot and long-term evaluation of a programme to help practitioners work more effectively with parents of babies and pre-school children to prevent childhood obesity. Child Care Health Dev 2010, 36(6):850-857.

35. Wen LM, Baur LA, Simpson JM, Rissel C, Wardle K, Flood VM: Effectiveness of home based early intervention on children's BMI at age 2: randomised controlled trial. BMJ 2012, 344:e3732.

doi:10.1186/2046-4053-2-28

Cite this article as: Lakshman et al.: Protocol for systematic reviews of determinants/correlates of obesity-related dietary and physical activity behaviors in young children (preschool 0 to 6 years): evidence mapping and syntheses. Systematic Reviews 2013 2:28.

\section{Submit your next manuscript to BioMed Central and take full advantage of:}

- Convenient online submission

- Thorough peer review

- No space constraints or color figure charges

- Immediate publication on acceptance

- Inclusion in PubMed, CAS, Scopus and Google Scholar

- Research which is freely available for redistribution 